\title{
Patterns of Technochange Management in ERP Multisite Implementations
}

\author{
A.Carugati, C. Gibson ${ }^{2}$, L. Mola ${ }^{3}$
}

\section{Introduction and theoretical framework}

Research and practices have focused on IT related project execution techniques for a long time and have produced very promising results in specifying methodologies for the inclusion and involvement of people and organizational factors into technical change processes. The methodologies that have had the biggest impact can be briefly resumed by the English born tradition of sociotechnical change (e.g $[1 ; 2])$ and the Scandinavian born tradition in participatory design (e.g. [3]). Despite the extreme value in IT project management of these techniques - and their derivates - there continue to be severe problems in getting business results from pervasive IT-related "technochanges". Technochange [4] refers to big, technologydriven, technology-dependent change seeking significant strategic benefits and requiring significant organizational change. From the management point of view these projects differ from smaller scale ones for their strategic dimension expressed in a need for alignment between technical and organizational changes and need for coordination across multiple projects active at the same time. The feeling is that while sociotechnical and participatory techniques are apt to confined projects - this can be evinced from the settings from which these techniques evolved - for technochange projects other techniques should be used. At the same time looking at which IT projects are undertaken by both large and medium corporations today we see a predominance of large scale projects like ERP implementations, BRP initiatives, integrations initiatives connected with mergers, etc. Sometimes the failure is acute, visible and public, as reported in the press: e.g. Socrate project in France [5], Taurus project at the London Stock Exchange in UK [6], more often the failure is chronic and may drag on and drag down business performance undetected for years. The importance of new techniques for project management is further underlined by the monetary amounts concerning these projects. A survey of cost structures for large scale projects suggests that the hardware and software costs are less than $20 \%$ of the total costs of implementation, small in comparison to installation and testing (45\%) and, most significantly, deployment or actually achieving

$1 \quad$ Aarhus School of Business - Denmark; ${ }^{2}$ MIT Sloan School of Management - USA; ${ }^{3}$ University of Verona - Italy 
effective use of technology (36\%). Douplaga and Astani [7] in a survey conducted among companies of various size to discovered the major issues concerning ERP implementations, show that the major problem for organizations of all sizes was the lack of ERP training and education. Moreover, according to SAP, the leader vendor in ERP industry, it is possible establish an average ratio of 1 to 5 between the costs for the software and the costs needs for consultancy, customizations and training.

This study focus therefore on the knowledge and training issues in multisite ERP implementations and proposes a contingent approach to implementation strategies in accordance to the level of knowledge possessed by the company in the ERP technology.

\section{Research Method}

This study is based on two cases of multisite ERP implementations in two large American and European enterprises. The companies are manufacturers and have been operating for many decades. These companies were chosen because of their similarities (both manufacturers, multinationals, long tenure) and because they have been going through major successful technochange efforts in the recent past. The choice of the company with multiple sites fits the needs of this study because it can be expected that these companies have gathered experience with ERP implementation over time. Another reason to chose these companies is that the American one decided to carry out the ERP implementation completely on their own while the European one decided to carry out the ERP implementation using consultants. This is interesting because similar observations will increase the external validity of the results. The case studies are based on observation and interview to highlight the similarities in activities and processes in the implementations of the two technochange projects. We focus the study on the history of the implementations starting from the reasons and then following the sequence of events, tactics, and methods applied until the end of the two projects. This method follows the practice based research carried out by Levina [9]. The data has been analyzed in order to identify major decision, methods and processes chosen. The results of this part will be presented in the case studies below. Then the cases have been analyzed to highlight recursion of practices. Finally the practices have been arranged in a sequence taking also input from existing theory.

\section{Case Study 1: Dow Corning Corporation}

Dow Corning (DC) was established in 1943 specifically to explore the potential of silicones. Today DC is a global leader in silicon-based technology, offering more than 7,000 products and services with the majority of annual sales outside the 
United States. In 1995 DC was in serious trouble, after fifty years of growth, the $\$ 2.2$ billion company was experiencing increasing global competition for its broad silicone-based product line. More pressing was the infamous breast implant situation since thousands of recipients were lining up for jury trials. With increasing pressure on earnings the CEO of DC led his operating committee through a strategic review. The business strategy that had evolved and served the company well was to be left intact but they hat to change the business processes and use IT as a significant enabler of change. Such a role for IT was new for DC that in that period was coming out of a large effort to create a global order entry system, a project called Goes - that resulted in a major technochange failure.

The executives assessed that the risk of success for IT-enabled operational change was very high. It was impossible for the analysts to get consensus among autonomous regional business units on systems requirements. While employees supported management in the current crisis situation, they had never experienced major changes. Management knew the DC culture was characterized by long job tenure and employee loyalty, but they had to make a case for transformational change. Management made two key decisions: the first was to appoint a new CIO in direct contact with the CEO; the second was the decision to accept the CIO recommendation to implement SAP R3 ERP.

The CIO called the change program "Project Pride". It unfolded in four distinct phases in 1996-1999. Each phase was characterized by different risks and DC management had to adapt different styles of project management. In phase one the CIO, in order to ensure employees would accept changes, decided not to use consultants, but to build capability and commitment having the work done in house. The CIO asked and received 40 of the best, most respected middle managers from operations around the world and made them the full-time implementation team. Few had IT experience, but they worked closely with IT. Employing a typical DC project management approach, consensus-oriented and with flexible milestones, the team began to learn SAP and to design work process changes to match SAP without modifying it.

Phase two of Project Pride began during the first year as the CIO reacted to what he saw as the limitations of the consensus-learning project style. While creative learning was certainly occurring, and the team of 40 became deeply committed to understanding SAP, little progress was made on redesigning processes. Employees in the field, aware of the executive pronouncements that big change was coming, were beginning to question the lack of firm milestones and signs of progress. The CIO took two important actions. First, he changed the project manager from one relatively comfortable with technology to a highly respected, result-oriented plant manager. Second, he tightened project planning to become more rigid: deadlines were set and expected to be met for a pilot implementation. At the same time he still left the Project Pride implementation team in charge of how they used their resources to meet the deadlines. This project approach led to a successful pilot implementation. The pilot was a full cutover to SAP for virtually all operations of a recently acquired autonomous business in Europe. The success 
of the pilot soon resonated throughout the DC culture as a symbol of top management determination and the capability of the project Pride team.

With the pilot done, the CIO recognized that he was in a new phase. There was a need for a change in project management to enable the worldwide implementation of SAP. The global scope and urgency of the project drove the risk and kept it high even though a climate of employee receptivity had been created. He modified the project style by strengthening the authoritative nature of his leadership and that of his lieutenants, while still permitting flexibility at the ground level. In the crucial period from 1997 into 1998, he led a relentless and unprecedented change effort at DC. He traveled extensively to spread the word and rally the project teams implementing SAP. He personally negotiated with and pressed his executive colleagues and old personal friends to adhere to their commitment to make changes. A key change came in 1998 when the CEO agreed to make project implementation one of the significant performance goals for the senior levels of line management. It was a strong statement of support for Project Pride.

At this point the fourth and final phase was underway. Although there were several pockets of reluctance, they were generally employees trying to maintain good customer relations and meet their operational goals: a positive form of resistance. The CIO and the teams picked up the pace and tightened and made rigid deadlines for site-specific sub-projects. Senior management stressed the new goals. Implementation time for sites went from 18 months after the pilot to 4 months in late 1998. In 1999 the installation of SAP was essentially completed. DC became the largest successful single-database installation of SAP R3 at that time, providing global integration for the company.

\section{Case Study 2: Gruppo Manni HP}

Gruppo Manni (GM) was established in the 1945 to recycle iron and steel. It was created by a single entrepreneur and developed its business by providing services to building yards becoming middleman between the steelworkers and final users. GM carries out industrial activities in steel-working division and in prefabricated steel elements, and components and structural systems for plants. They have a revenue of $492 \mathrm{m€} /$ year and employ 800 employees in 8 operative companies and 15 production and distribution centers.

In the 1996 the CEO became aware that the growth of the group, in value and volumes was not supported enough by the existing IT. High costs of maintenance, due to the high customization; functional bugs and architectural limits were some of the aspect that worried GM managers. In order to identify the weaknesses of the information systems a task force was created composed by the CIO, the project manager who developed the system in use and two external IT consultants. These employees founded together with GM a consulting company called Ratio with the specific purpose to aid the technochange initiative. 
A first assessment underlined the emergent needs of the group: centralization of the business decision processes common to the whole company; establishment of operational standard and common procedures among all the companies belonging to the group; optimization of marketing tools; integration with suppliers; real time connection among all the companies of the group; centralization and standardization of the IT infrastructure. The solution found for all these problems was to implement a group wide ERP which could cover all core and support processes. Ratio consultant decided, given the requirements identified to adopt a multisite ERP, called Diapason developed by Gruppo Formula.

Ratio consultants decided for a gradual rollout per module and per site. They also went into a partnership with Gruppo Formula for the implementation project. Ratio personnel was involved in Formula's ERP implementation Project as observers in order to create a Ratio's internal group of consultants able to run a ERP implementation project from the both the technical and the managerial side. The initial phase while involving consultants had the same goal as in DC: learning first in the single site module by module and then move to multiple sites. They decided to implement the ERP system modules starting with finance followed by sales, manufacturing, and procurement.

The original timetable of the gradual rollout project was as follow: September '97 Project start up; January 98 Finance in the Holding Go live; April '98 Controlling in the Holding GO live; January '99 Sale, Procurement and Manufacturing Go live in 2 Companies of the group as pilots; January '00 Procurement and Manufacturing Go live in all the company of GM. During 1998 in response to the successful implementation of the finance and controlling modules the CEO and CIO of GM asked for a profound change in the philosophy of the project. Rather than a gradual rollout, as originally planned, they asked for a "big bang" approach for the Sales, Manufacturing and Procurement modules in all sites. In January and February 1999 the tuning phase involved all personnel of GM.

\section{Framework development}

The implementation of the global order entry system (Goes) and ERP (Pride) in the DC and the analysis and design in GM can be distilled to a cyclical process of strategy setting, risk assessment, and choice of appropriate execution style. All this activities appear, in the two companies to be centered on a relentless search to find in each situation the right learning style to decrease risk. This might not be evident at first glance but from the passage from risk assessment to execution there is an explicit or implicit effort to increase learning. The learning issue appears more clearly in phase one where the effort - learning ERP (SAP R3 for DC and Diapason for GM) with a limited use of external consultants - is explicit but returns in the other phases as well when the pilot projects are used to show - i.e. teach - the company ability to pull of these kinds of projects. This is a key ele- 
ment in multisite ERP implementations because after the first implementation there is the need to convince others to follow suit. In other words the top managers at DC and GM were intuitively mindful that strategic direction, risk and learning style and execution style where tightly coupled and their accurate choice was key to success. In each phase they implicitly or explicitly adjusted their strategy, conducted change risk assessments, used different learning styles to mitigate risks, and adjusted the method of project management to cope with remaining risk. The path followed by the projects in DC and MG can be seen in figure 1 .
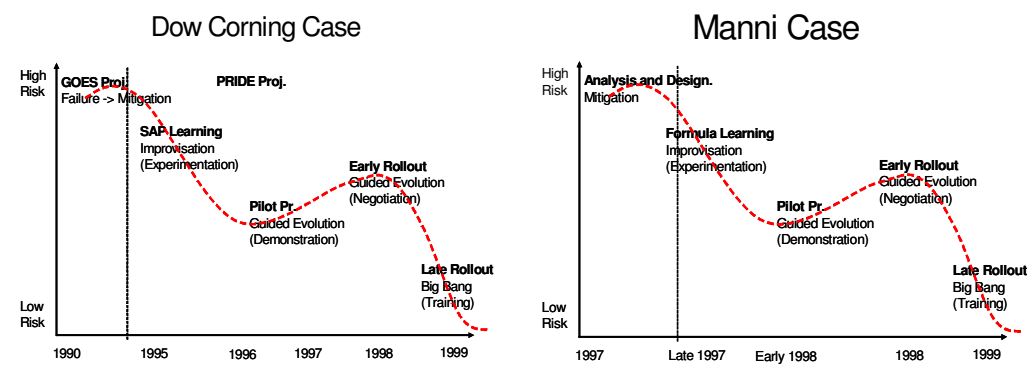

Figure 1. Risk profile and management/learning style adaptation at DC and GM

An interesting observation is that in the two companies they seemed to have grasped the concept that risk is not an absolute value. What is risky - or in other words difficult - can become straightforward when you know enough about the problem. By using different learning styles they were able to mitigate risks not by changing the nature of the problem but by changing their approach to it.

The second observation is that throughout the Pride project there was a continuous active participation of the highest levels of management. This seems to be necessary and mandatory for technochange processes. Top management involvement is necessary because of the large array of methods used. Only top management could accept the slow pace of the first phase of experimentation in the same way that only top management could change the reward structure to include project implementation in the performance goals for the senior levels of line management. Finally only top management could use the iron fist in the latest phase of the project when SAP was rolled out with a Big Bang style at record speed.

A third interesting point is to observe the wave pattern of the Pride project. Risk is not always decreasing because the nature of the problem changes. In 1998 the CIO had to engage in complex negotiations to assure the buy-in of the other sites in view of a smoother implementation later on. This part of the project quite typical of technochange - is very different from before but could not be carried out without the knowledge previously created. The strong knowledge base obtained in phase one and two provided solid arguments that reasoned well with the local plant managers and line managers. In this delicate phase the CIO adopted a Top-down Coordination method of project management, with an authoritative 
style accompanying his traveling and convincing, but allowing for flexibility in timetables for particular projects.

Summarizing the observation of the DC case can be translated into a framework for the execution of technochange processes. The framework focus on the three macro activities: strategy setting, risk assessment and execution style, and conscious management of knowledge. The framework presents three phases, connected by guidelines for assessment and use. The three frameworks and guidelines are three steps in an iterative process for assessment and execution.

First, providing and understanding of the strategy, vision and context for programs and projects and checking progress of the program and project in their contexts over time. In this step initially the business case for the technochange program or project is created: covering the specific metrics of intended business success and the broad outline of the technical solution. We refer to these as the direct business technochange efforts: achieving cost savings, creating new revenueproducing service offerings, meeting a mandated requirement such as $\mathrm{Y} 2 \mathrm{~K}$, or other business results. This first step also advocates separating these direct types of programs and projects from indirect efforts. Indirect programs or projects are those aimed at enhancing the capability of the enterprise. Indirect activities may include such goals as achieving an enterprise-wide IT architecture, changing the work culture, creating a learning organization, and the like. The business case in this step leads to a specification of the nature of the project in terms of its inherent difficulty, apart from the organization's capability to succeed with it.

Second, conducting a diagnosis of the capability to carry out the particular technochange program defined in step one. Given the description and status of the program/project from step one, this step assesses how well leaders and stakeholders can build and install technology, make organizational changes, and address problems of alignment and coordination. The sequence in this step is a risk assessment of these capabilities, then a classification of the type of "learning" required to deal with the contextual level of risk, the approach to learning, and the nature of the execution. Taking inspiration from the DC and the GM cases and from Gibson [10], we offer and explain three styles of program and project management for execution. For high risk and experimental learning, an improvisational approach; for low risk and straightforward learning (such as traditional "training"), a "big bang" approach; and for moderate risk and for demonstrative or negotiated learning, either an "evolutionary" or a "coordinated" approach.

Third, execution of the approach using appropriate techniques and mechanisms. For example, an improvisational approach will typically require mechanism to support intensive, focused, experimental learning by key individual stakeholders such as users of a new system or developers using a new technology. On the other hand a big bang approach could require mechanisms for widespread organizational communications and elaborate planning for coordinated cutover.

The three steps are repeated iteratively, with each cycle ending up with a review from the strategic level based on the success and issues with the previous execution phase. In technochange the reviews are an important component with 
new risk assessments as the effort progresses, and changes in the execution approach as required. The way in which evaluation takes place is shown in figure 2 .

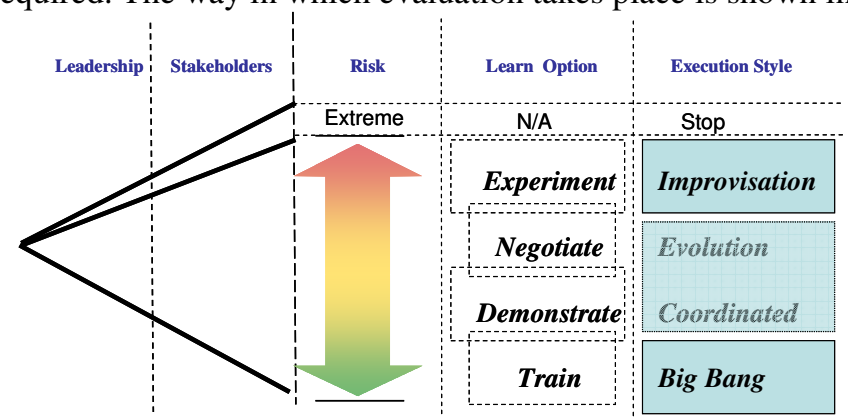

Figure 2. Framework for Technochange Execution

The diagnosis phase is carried out investigating the capability of the company leadership and of the other stakeholders. If both leadership and stakeholders have proven capabilities to carry out a given technochange process then the process is low risk and the learning required is of direct transfer of information - training like. If both leadership and stakeholders are incapable of carrying out such change then the risk is extreme and the options are either to redefine the problem differently or to engage in intensive learning of the experimental kind as carried out at DC in the first phase of Pride project. In case of mixed capability we end up in a grey zone where negotiation is required. We have put the leadership to the left giving it more importance in driving the risk factor because both in the DC and in the GM cases the type of decisions related to technochanges required first of all leadership understanding.

\section{References}

1. Mumford E., 2003, Redesigning Human Systems, Idea Publishing Group

2. Checkland P., Scholes J., 1999, Soft Systems Methodology in Action, Wiley, Chichester.

3. Mathiassen L., 1999, http://www.cs.auc.dk/ larsm/rsd.html (visited in February 2003)

4. M.L. Markus, Technochange management: Using IT to drive organizational change. Journal of Information Technology. Volume 19, pp.4-20, (2004)

5. Mitev, N.,N., (1996) More than failure? The computerize reservation system at France railways Information Technology and People v.9 n.4 pp. 8-19

6. Drummond, H., (1998) Riding a Tiger: Some lessons of Taurus, Management Decision, v36, n.3 pp.141-146

7. Duplaga, E.A., \& Astani, M. (2003) Implementing ERP in Manufacturing, Information System Management, v20 n.3, pp. 68-75.

8. Yin, R.,K., (1994) Case Study Research: Design and Methods, 2nd ed., Vol. 5 Thousand Oaks, CA: Sage Publications Inc.

9. Levina, N. (2005), Collaborating on Multiparty Information Systems Development Projects: A Collective Reflection in Action View, Information Systems Research, Vol. 16, No. 2, Pp. 109-130.

10. Gibson C, 2003, IT-enabled Business Change: An Approach to Understanding and Managing Risk, MISQ Executive, September 2003, Vol. 2, No. 2 